\title{
Gut microbiota manipulation with prebiotics in patients with non-alcoholic fatty liver disease: a randomized controlled trial protocol
}

\author{
Jennifer E. Lambert ${ }^{1}$, Jill A. Parnell ${ }^{2}$, Bertus Eksteen ${ }^{3,4}$, Maitreyi Raman ${ }^{4}$, Marc R. Bomhof ${ }^{1}$, Kevin P. Rioux ${ }^{4,5}$,
} Karen L. Madsen ${ }^{6}$ and Raylene A. Reimer ${ }^{1,7^{*}}$

\begin{abstract}
Background: Evidence for the role of the gut microbiome in the pathogenesis of non-alcoholic fatty liver disease (NAFLD) is emerging. Strategies to manipulate the gut microbiota towards a healthier community structure are actively being investigated. Based on their ability to favorably modulate the gut microbiota, prebiotics may provide an inexpensive yet effective dietary treatment for NAFLD. Additionally, prebiotics have established benefits for glucose control and potentially weight control, both advantageous in managing fatty liver disease. Our objective is to evaluate the effects of prebiotic supplementation, adjunct to those achieved with diet-induced weight loss, on heptic injury and liver fat, the gut microbiota, inflammation, glucose tolerance, and satiety in patients with NAFLD.

Methods/design: In a double blind, placebo controlled, parallel group study, adults (BMI 225 ) with confirmed NAFLD will be randomized to either a $16 \mathrm{~g} / \mathrm{d}$ prebiotic supplemented group or isocaloric placebo group for 24 weeks ( $n=30 /$ group). All participants will receive individualized dietary counseling sessions with a registered dietitian to achieve $10 \%$ weight loss. Primary outcome measures include change in hepatic injury (fibrosis and inflammation) and liver fat. Secondary outcomes include change in body composition, appetite and dietary adherence, glycemic and insulinemic responses and inflammatory cytokines. Mechanisms related to prebiotic-induced changes in gut microbiota (shot-gun sequencing) and their metabolic by-products (volatile organic compounds) and de novo lipogenesis (using deuterium incorporation) will also be investigated.
\end{abstract}

Discussion: There are currently no medications or surgical procedures approved for the treatment of NAFLD and weight loss via lifestyle modification remains the cornerstone of current care recommendations. Given that prebiotics target multiple metabolic impairments associated with NAFLD, investigating their ability to modulate the gut microbiota and hepatic health in patients with NAFLD is warranted.

Trial registration: ClinicalTrials.gov (NCT02568605) Registered 30 September 2015

Keywords: Prebiotic fiber, Gut microbiota, Fatty liver, Weight loss, Fibrosis

\footnotetext{
* Correspondence: reimer@ucalgary.ca

${ }^{1}$ Faculty of Kinesiology, University of Calgary, 2500 University Dr. NW, Calgary,

AB T2N 1N4, Canada

${ }^{7}$ Department of Biochemistry \& Molecular Biology, Cumming School of

Medicine, 3330 Hospital Drive NW, Calgary, AB T2N 4N1, Canada

Full list of author information is available at the end of the article
} 


\section{Background}

Non-alcoholic fatty liver disease (NAFLD) is now the most common cause of chronic liver disease worldwide [1]. NAFLD can progress from simple steatosis to nonalcoholic steatohepatitis (NASH) and finally to cirrhosis and its complications (e.g. hepatocellular carcinoma) [1]. Currently the prevalence of NAFLD is $20-30 \%$ of the general population in affluent countries [2-4]. Since NAFLD is a pathological condition closely associated with obesity and insulin resistance [5], it could be expected that rates will continue to rise in conjunction with the growing severity of obesity [6].

\section{NAFLD Pathogenesis}

In 1998, Day and James [7] proposed a 'two hit' hypothesis to describe the pathogenesis of NAFLD whereby insulin resistance [8] contributes to steatosis (first hit), which sensitizes the liver to oxidative stress (second hit) leading to inflammation, fibrosis and necrosis. Additional theories, such as the multiple-hit hypothesis, suggest factors including adipokines and mitochondrial dysfunction may also contribute to NAFLD [9]. Unrestrained free fatty acid flux from adipose tissue has been implicated as a primary contributor to steatosis $[10,11]$, however recent work has indicated that de novo lipogenesis is significantly elevated in NAFLD patients and contributes to both plasma and hepatic fatty acid levels [12-14].

The prevalence of NAFLD is estimated to be as high as $75 \%$ in individuals with obesity, $93 \%$ in individuals with morbid obesity, and 47-87 \% in people with type 2 diabetes [1]. From a clinical perspective, it is not the simple steatosis that is of concern, but rather the progression to NASH and fibrosis that carries risk for morbidity and mortality [15]. Among individuals with NAFLD, $10 \%$ will progress to NASH, and a third of those patients with early-stage NASH will progress to cirrhosis within 5-10 years, putting tremendous strain on liver transplant registries [16]. In the United States, there was an 8-fold increase in liver transplants attributed to NAFLD between 2001 and 2009 [17]. Aside from hepatic damage, steatosis may accelerate the progression of dyslipidemia, insulin resistance, and atherosclerosis [18], as well as increase the risk for cardiovascular [19] and kidney disease [20]. Given the increased pressure on already over-burdened health care systems, this patient population represents a group in need of effective treatments. As yet, there is a lack of approved treatments for patients with NAFLD, particularly with fibrosis, and therefore investigation of promising targets, including dietary interventions, are critically needed.

\section{NAFLD Treatment}

Currently there are no medications or surgical procedures approved for the treatment of NAFLD. Several agents have been tested, including vitamin $\mathrm{E}$ and the thiazolidinediones class of diabetes medications [21-24], though limitations have been identified with both. In the PIVENS (Pioglitazone, Vitamin E, or Placebo for NASH) trial, vitamin $\mathrm{E}$ and pioglitazone both improved hepatic steatosis and lobular inflammation but not fibrosis, and pioglitazone was associated with weight gain ( $4.8 \%$ increase in body weight) [22, 25]. Rosiglitazone improved steatosis but not any other histologic lesions in patients with $\mathrm{NASH}$, and was also associated with weight gain [21].

Weight loss via lifestyle modification remains the foundation for current clinical management of the disease $[26,27]$. A reduction in weight of 3-5\% improves biochemical markers and steatosis in NAFLD patients while $10 \%$ weight loss is required for improvement in inflammation and NASH regression [28, 29]. Still, identification of effective and high impact lifestyle interventions to achieve this degree of weight loss is critically needed to provide evidence-informed recommendations.

\section{Gut microbiota in NAFLD, obesity and associated co-morbidities}

Gut microbiota have emerged as an important environmental factor influencing the pathogenesis of NAFLD $[9,30]$. Since the liver and intestine are connected anatomically and via the hepatic portal system, the gut microbiota and their metabolic by-products may influence hepatic pathology. The connection between the gut microbiota and NAFLD is incompletely understood, however, and a recent review only identified four animal and five human studies characterising microbial profiles in NAFLD [31]. In mice fed a high fat diet, Lactobacillus positively correlated with the severity of hepatic steatosis and the effect was attributed to the impact of Lactobacillus on bile acid metabolism [32]. Supplementing a high-fat diet with Bifidobacterium pseudocatenulatum CECT 7765 over seven weeks increased Bifidobacterium and decreased Enterobacteriaceae; changes associated with a reduction in hepatic steatosis in conjunction with weight loss and improved insulin sensitivity [33]. Finally, inflammasome-deficient mice displayed a dysbiotic microbiome that exacerbated hepatic steatosis and inflammation [34].

In humans, using experimental dietary choline depletion (known to induce hepatic steatosis), higher baseline levels of Gammaproteobacteria correlated with a lower risk for fatty liver. Conversely, Erysipelotrichi correlated with a higher risk, suggesting a person's microbiome can impact their susceptibility to fatty liver in response to choline deficiency [35]. Quantification of gut microbiota in NAFLD patients has produced conflicting results [36-38] and differences within phyla highlight the need for deeper sequencing. Furthermore, in many studies the effects of NAFLD were not isolated from those of simple 
obesity. From a treatment perspective, administration of a probiotic combination increased Bacteroidetes and decreased Firmicutes after six months, which correlated with reduced hepatic fat [39]. Further, a meta-analysis evaluating probiotic treatment in NAFLD, found reduced liver enzymes (ALT, AST), TNF- $\alpha$, total cholesterol and insulin resistance, suggesting that altering gut microbiota may beneficially affect NAFLD [40].

Changes in the gut microbial environment in obesity and type 2 diabetes have been well-documented. These conditions, which are closely tied to NAFLD development, are known to be associated with changes in the vast and diverse communities of microorganisms that live in the human intestinal tract [41-44]. Although not unanimously shown, obesity is associated with a phylum-wide increase in Firmicutes and a reduction in Bacteroidetes [42, 45-47]. This shift is believed to enhance energy harvest, thereby promoting weight gain, hyperglycemia and insulinemia [43, 48-50]. The microbiota also affect the integrity of the gut barrier; in the case of obesity and high fat diets, changes in gut integrity translate to increased intestinal permeability and translocation of endotoxin (lipopolysaccharide from Gram-negative bacteria) [9, 51-53]. Increased leakage of endotoxin into the bloodstream is called metabolic endotoxemia and has been linked to the onset of metabolic diseases [52]. In NAFLD patients, endotoxin levels are elevated $[54,55]$ and have been associated with small intestinal bacterial overgrowth $[9,56,57]$. The microbiota can also produce endogenous ethanol [9] and 300 other volatile organic compounds (VOC) [58], some of which may contribute to liver injury. Recently, it has been shown that obese NAFLD patients have an altered microbiome and unique fecal VOC profile compared to healthy controls [38]. While the effect of VOC on liver function is unknown, it is plausible that excess VOC may contribute to a state of elevated inflammation and hepatocyte stress. Given the profound metabolic impact of the gut microbiota to the host, therapeutic manipulation of this community may aid in treatment of NAFLD.

\section{Prebiotics}

Prebiotics are nondigestible food ingredients that are fermented in the gut and modulate the microbiota in a manner beneficial to the host [59-61]. Prebiotic fiber is found naturally in common foods such as asparagus, garlic, leeks and onions [62]. The average intake of prebiotic fiber is estimated to be $1-4 \mathrm{~g} / \mathrm{d}$ in the Unites States and 3-11 g/d in Europe [63].

There are numerous animal and human studies that point to the potential of prebiotics to treat NAFLD $[60,64-67]$. Our lab and others have consistently shown that prebiotic supplementation is associated with lower body weight and/or reduced weight gain in normal
[68], genetically obese [69] and high fat, high sucrose-fed $[70,71]$ rats. We were also the first to report that prebiotic supplementation improves weight loss and reduces energy intake in overweight and obese adults compared to placebo [72], which has since been shown in patients with type 2 diabetes [73]. Increases in satiety hormones observed with prebiotics, including glucagon-like peptide-1 (GLP-1) and peptide YY (PYY) and decreases in the orexigenic hormone ghrelin, likely mediate some of the reduced ad libitum energy intake contributing to weight loss [68, 70-72, 74, 75]. These modifications observed with prebiotic supplementation are notable because greater satiety and lower hunger are associated with improved adherence to weight loss interventions [76-79]. Further, the metabolic benefits of prebiotics were evaluated in a recent systematic review of 26 randomized controlled trials [80], where prebiotics were shown to improve satiety, postprandial glucose and insulin in both healthy and obese individuals.

Prebiotics alter gut microbiota in favor of host health [63], including modifying gut barrier integrity and endotoxin translocation. For example, prebiotics increase Bifidobacterium which is correlated with lower serum endotoxin levels [81]. In addition, prebiotics stimulate the gut trophic hormone glucagon-like peptide-2, which can modulate endotoxin translocation via effects on epithelial tight junctions [53]. Steatosis itself increases the vulnerability of the liver to injury from endotoxin; while other NAFLD treatment such as vitamin E and thiazolidinediones may reduce steatosis, there is no human evidence that currently suggests vitamin $\mathrm{E}$ or the glitazones attenuate endotoxin levels.

The NAFLD Activity Score (NAS) is a histologicallybased tool used to grade steatosis, lobular inflammation and ballooning in patients with NAFLD. While weight loss has been shown to reduce NAS, this is largely driven by reductions in steatosis but not ballooning or inflammation, and it does not appear to affect fibrosis [82]. By contrast, we have preliminary histopathological evidence from a pilot trial suggesting that prebiotic fiber improves several hepatic outcomes (i.e. fibrosis) that would augment improvements from weight loss alone (i.e. steatosis) (Unpublished observations; RA Reimer, MR Bomhof). This finding is supported by a recent report that 24 weeks of prebiotic (fructooligosaccharide) plus probiotic (Bifidobacterium longum) reduced serum AST, endotoxin and hepatic steatosis in NASH patients [83]. Our recent review [61] highlights convincing evidence from animal and human studies for the potential of prebiotics as a multi-target strategy to treat NAFLD. It is our hypothesis that prebiotic supplementation, as an adjunct to lifestyle-mediated weight loss, will improve hepatic and metabolic pathways beyond those targeted by weight loss alone. 


\section{Specific objectives}

The primary objective of this study is to determine the efficacy of a prebiotic supplement in combination with registered dietitian supported weight loss in reducing hepatic injury and liver fat in NAFLD patients over 6 months. Secondary objectives include determining the effect of prebiotic supplementation plus weight loss on appetite, body composition, glucose tolerance, inflammatory cytokines, and potential mechanisms related to gut microbiota, volatile organic compounds, and de novo lipogenesis. The research study is novel in that it has been designed to provide comprehensive information on liver health outcomes in NAFLD patients in conjunction with a mechanistic investigation into several of the "multi-hits" thought to promote NAFLD and its progression to NASH.

Given the primary objective of determining the impact of prebiotics on hepatic injury and liver fat in NAFLD patients from baseline to 6 months, patients will undergo a liver MRI, a FibroScan and blood FibroTest. Changes in gut microbiota, VOCs, and LPS will elucidate the effects of prebiotic modulation of the gut microbiota and their metabolic by-products. The impact of prebiotics on obesity-related outcomes will be evaluated through anthropometrics, subjective appetite ratings and satiety hormone responses. Insulin resistance is also closely associated with NAFLD and the effects of prebiotics on glucose regulation will be assessed via responses to an oral glucose challenge. Inflammatory cytokines are another potential "hit" and the effect of prebiotics on plasma inflammatory markers will be quantified. Finally, there is potential for prebiotics to reduce lipogenesis due to its effects on glucose tolerance, food intake, and gut microbiota, and this will be investigated via measurement of de novo lipogenesis. This trial is designed to test whether prebiotic supplementation augments the reduction in steatosis attributed to weight loss alone. We hypothesize that the addition of a prebiotic supplement to a registered dietitian-led weight loss intervention, will improve hepatic injury and steatosis to a greater extent than weight loss alone.

\section{Methods/design}

\section{Ethics, consent and permission}

This proposal has been approved (REB14-2464) by the Conjoint Health Research Ethics Board of the University of Calgary (Calgary, AB, Canada). Voluntary, written, informed consent will be obtained from each participant.

\section{Design}

The study is a single-center double blind, placebo controlled, parallel group study. Patients will be randomized to a $16 \mathrm{~g} / \mathrm{d}$ prebiotic supplemented group or isocaloric placebo group for 24 weeks. All participants will receive individualized dietary counseling sessions with a registered dietitian to achieve $10 \%$ weight loss.

\section{Inclusion criteria}

Adult participants (aged 18-65, BMI $\geq 25 \mathrm{~kg} / \mathrm{m}^{2}$ ) diagnosed with NAFLD on the basis of abnormal liver enzymes (ALT > 1.5x upper limit of normal) and ultrasonography [84] will be recruited from the South Health Campus Fatty Liver Clinic and the surrounding community in Calgary, Alberta, Canada. Individuals with type 2 diabetes treated with diet and exercise alone or metformin will be included, as well as individuals with aspartate aminotransferase and alanine aminotransferase $\leq 10 x$ upper limit of normal.

\section{Exclusion criteria}

Exclusion criteria include other causes of liver disease such as viral hepatitis and alcoholic liver disease; cirrhosis of the liver (FibroScan $>17.5 \mathrm{kPa}$ or FibroTest $>0.8$ ) or clinical features of cirrhosis; alcohol consumption $>20 \mathrm{~g} /$ day (2 standard drinks) in women or $>30 \mathrm{~g} / \mathrm{d}$ ( 3 drinks) in men; alternate (e.g. TPN) or concomitant etiology for abnormal liver enzymes; history of decompensated liver disease including ascites, encephalopathy or variceal bleeding; concomitant use of any weight loss medication or herbal weight loss products, previous bariatric or other intestinal surgery known to affect food intake or digestive function; presence of active infection, pregnancy or lactation; regular use of a probiotic or prebiotic supplement within 3 months prior to enrollment; antibiotic use within 3 months prior to enrollment; weight loss $>3 \mathrm{~kg}$ within the preceding 3 months to enrollment; uncontrolled cardiovascular or respiratory disease, active malignancy, or chronic infections; use of agents such as vitamin E, omega-3 fatty acids or medications with evidence for effects on NAFLD (pioglitazone, GLP-1 analogues, dipeptidyl peptidase IV inhibitors, ursodeoxycholic acid); and patients with type 2 diabetes where $\mathrm{HbA} 1 \mathrm{c}$ is $>9 \%$.

\section{Randomization}

Participants will be randomized in equal numbers to two groups, stratified by sex, BMI, and age for allocation into prebiotic or placebo arms. Randomization sequences will be prepared by an independent statistician and entered into a web-based central randomization program. Sequences will not be revealed to investigators or study staff. Randomization will be in blocks of 4 within each sex/age/BMI stratum. To maintain blinding of the trial coordinator and registered dietitian, a research assistant with no involvement in the study will use the central randomization program at the randomization visit. Study personnel will be unaware of treatment 
allocation prior to the assignment of interventions, using sequentially numbered, opaque, sealed envelopes to maintain allocation concealment. The clinical trial coordinator will enroll participants. Participants, research staff and outcome assessors will be blinded to participants' assigned group. Participants will receive the prebiotic or placebo supplement in identical foil packets to maintain blinding. An overview of the study design and measurement outcomes is presented in Fig. 1.

Participants will be randomly assigned to a $16 \mathrm{~g} /$ day prebiotic dose [Synergy1 (oligofructose-enriched inulin),
Beneo-Orafti, Belgium] or an equicaloric dose of placebo [maltodextrin] for 24 weeks. The dose will be ramped up over two weeks and at full dose be consumed as two $8 \mathrm{~g}$ packets/day (13.2 kcal/packet) or two $3.3 \mathrm{~g}$ packets/day (13.2 kcal/packet) of maltodextrin. Participants will add the packet to $250 \mathrm{ml}$ of water and consume it 30 minutes prior to lunch and dinner. The dose of $16 \mathrm{~g} / \mathrm{d}$ was selected given previous work showing a reduction in serum AST in NASH patients with prebiotic [85]. Compliance will be assessed via packet counts.

\section{A}

\section{Dietary Intervention Study Design}

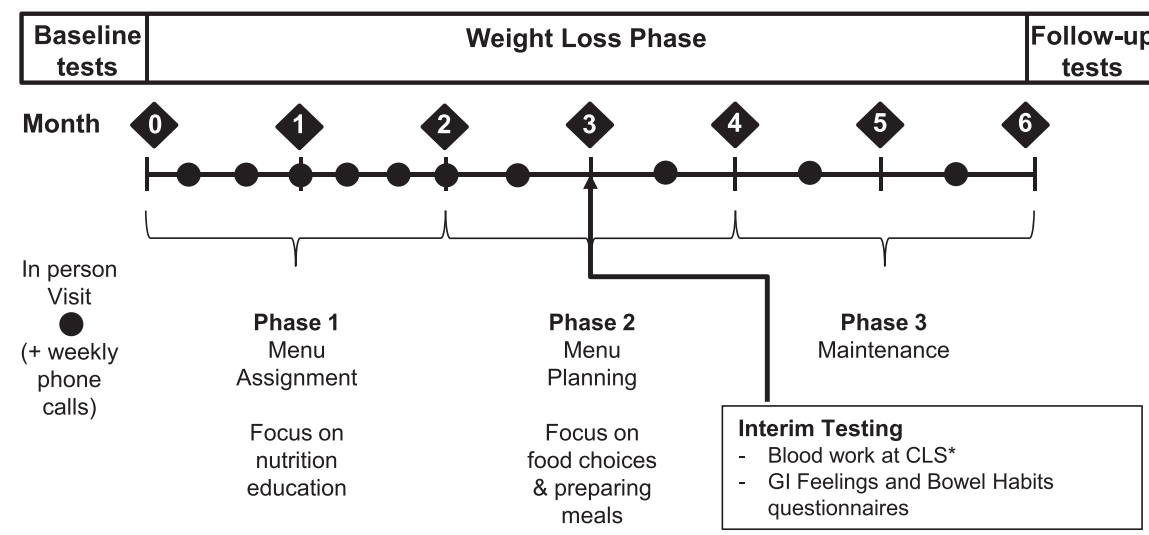

Body weight and waist circumference measured at all visits;

3-Day Food Records completed at 0,4,10,16, 24 weeks; VAS measured weekly

\section{B}

\section{Baseline and Follow-Up Testing Protocol}

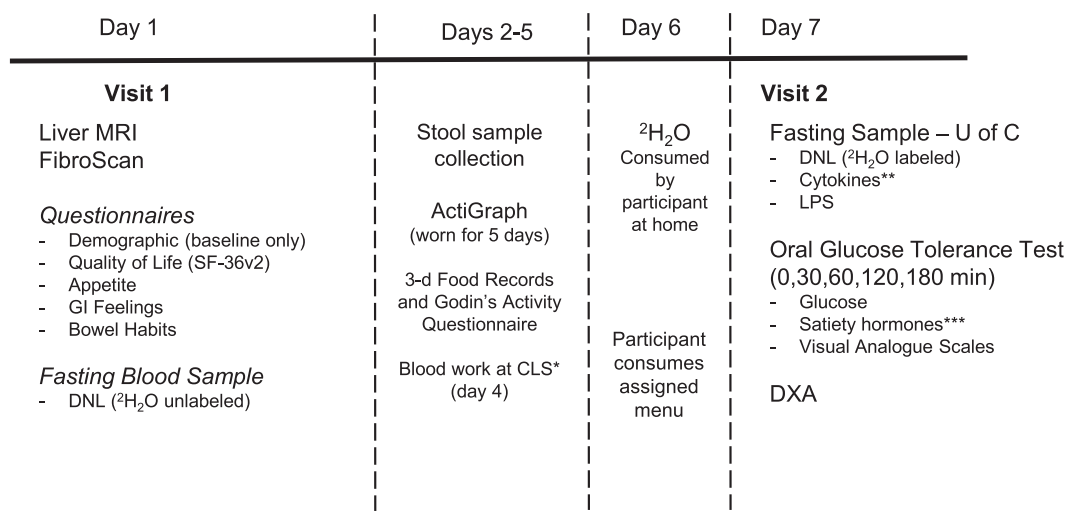

Fig. 1 Schematic overviews of the a study design and $\mathbf{b}$ testing day protocols. *Blood work at Calgary Laboratory Services (CLS) includes: glucose, lipids (total, LDL, HDL, TG), HbA1C, liver enzymes (ALT, AST, ALP), FibroTest (gamma-glutamyl transpeptidase, bilirubin, a2-macroglobulin, haptoglobin, and apo-lipoprotein A1), CRP, and liver function biochemistries (creatinine, electrolytes, albumin, bilirubin). ${ }^{* *}$ Adipokines/Cytokines include: adiponectin, MCP-1, TNF-a, IL-6 and IL-8. ***Satiety hormones include: active ghrelin, insulin, leptin, total GIP, active GLP-1, and total PYY 


\section{Dietary weight loss intervention}

All participants will be provided diet and lifestyle counseling in one-on-one sessions with a registered dietitian. Participants will be responsible for buying and preparing their own food. The diet will be designed to achieve a weight loss of $0.5-1.0 \mathrm{~kg} /$ week with an appropriate energy deficit based on anthropometrics and indirect calorimetry analysis. Participants will meet with the dietitian for 10 in-person visits (1-2 h each). Nutrition education and behavior modification strategies will be used to reduce intake of total energy, added sugars, sodium, and processed food, and to maintain adequate protein intake. Dietary intake during the phases will be assessed by weighed 3-day food records. The dietitian will also contact participants by phone each week to assess adherence, address questions or issues that arise, and monitor body weight. Body weight and waist and hip circumferences will be measured at each visit, and there will be a fasting blood draw at the midpoint of the study (week 12) to monitor blood chemistries (serum aminotransferases and lipids).

During the first phase (visits 1-4), participants will be provided with two 7-day meal plans designed by the dietitian. Meal plans will be personalized for each participants, taking into consideration lifestyle and food preferences based on questionnaires completed at screening. Nutrition education components will include identifying primary food sources of fat, carbohydrate, and protein, reading nutrition labels, and sources of empty calories. In the second phase (visits 5-8), the focus will be on behavior modification and achieving dietary autonomy. This will be achieved through specific counseling which will include planning and preparing meals in the home, portion control, and choosing foods when dining out. Counseling will also be tailored to each individual, based on initial questionnaires related to food craving and behavior. During the final phase (visits 9 and 10), participants will be entirely responsible for planning their own meals and counseling will focus on maintenance of dietary behaviors.

\section{Primary outcome}

\section{Hepatic injury and liver fat}

The primary outcome is change in hepatic injury and liver fat from baseline to 24 weeks. Improvement in liver injury will be defined by reduction in noninvasive and biochemical markers of fibrosis and inflammation. Fibrosis will be measured at baseline and 24 weeks using transient elastography (FibroScan), which has a high sensitivity for diagnosis and prognostic stratification of varying degrees of liver fibrosis [86, 87] and the Fibrotest which is a composite of serological markers and age and sex, that provides a quantitative estimate of liver damage (age, sex, gamma-glutamyl transpeptidase, bilirubin, $\alpha 2$-macroglobulin, haptoglobin, and apo-lipoprotein A1) [88]. The newer XL probe, specifically designed for obese patients, will be used for FibroScan [86]. Magnetic resonance imaging (MRI) will be performed at baseline and 24 weeks on a 1.5 T scanner (GE Medical Systems, Milwaukee, USA) to assess change in liver fat. MRI can detect fat in microscopic quantities and with chemical shift imaging (CSI) has a sensitivity of $90 \%$ and specificity of $91 \%$ [89]. Although costly, MRI is a front-runner in imaging modalities to quantify hepatic steatosis and represents an acceptable alternative to invasive liver biopsy. While still the gold standard in histological assessment of NAFLD, biopsy is not without risk and is limited by its lack of representation of the liver as a whole [90].

\section{Secondary outcomes}

Anthropometrics and Dual Energy X-Ray Absorptiometry

Height will be measured at the beginning of the study to confirm BMI. At each dietitian visit, body weight will be measured using a calibrated balance beam scale along with waist circumference and blood pressure. Body composition will be assessed at baseline and week 24 by DXA (Hologic QDR 4500, Hologic, Inc., Bedford, MA).

\section{Food record}

Food and beverage intake will be assessed using 3-day weighed food records at baseline, 4, 10, 16 and 24 weeks using food scales and standardized forms provided to the participants. Dietary adherence will be defined as prescribed versus measured energy intake. Prior to the start of the study all participants will attend a training session delivered by the dietitian in which they will be instructed on the use of the food scale and how to record their food intake. Participants will then be instructed to weigh and record all food consumed for 2 weekdays and 1 weekend day. This information will be analyzed with FoodWorks software (The Nutrition Company, Long Valley, NJ). Food records will also be used to assess dietary adherence.

\section{Physical activity and sleep monitoring}

Participants will be instructed to maintain their current level of physical activity throughout the study. Physical activity will be monitored at 0,12 , and 24 weeks using a modified Godin's Leisure Time Exercise Questionnaire that includes duration [91]. Objective monitoring of sleep and physical activity will occur at baseline and 24 weeks. Participants will be provided with an ActiGraph wGT3X-BT (ActiGraph, Pensacola, FL) monitor initialised at $30 \mathrm{~Hz}$ to wear for five consecutive days. Participants will be instructed to wear the monitor on 
their waist during waking hours (except when in contact with water) and on their wrist while sleeping, as the monitor has been validated in these positions [92]. At least 3 days of valid wear will be required for the data to be included in the analysis [93]. ActiLife6 software (ActiGraph, Pensacola, FL) will be used to convert data to 1-minute epochs. For physical activity, counts per minute will be classified using predetermined tri-axial vector magnitude cut-points for light, moderate, hard or very hard 0-2690, 2691-6166, 6167-9642, and >9642 counts per min respectively [94]. Sleep will be scored using the Cole Kripke algorithm [95] included in the ActiLife software.

\section{Subjective and objective assessments of appetite}

Subjective ratings of appetite will be recorded by participants at home following a meal, at the same time on the same day, each week using a validated $100 \mathrm{~mm}$ VAS $[96,97]$. The questions asked based on how the participant felt over the past week are:

1) "How hungry do you feel?" anchored by "I am not hungry at all" and "I have never been more hungry".

2) "How satisfied do you feel?" anchored by "I am completely empty" and "I cannot eat another bite".

3) "How strong is your desire to eat" anchored by "I have no desire to eat" and "I have a great desire to eat".

4) "How full do you feel?" anchored by "Not at all full" and "Totally full".

5) "How much do you think you could eat?" anchored by "Nothing at all" and "A lot".

6) Over the course of the past week, my appetite was: "Much lower than usual" and "Much higher than usual".

\section{Quality of life and gastrointestinal feelings}

Quality of life will be measured at baseline and 24 weeks with the SF-36v2 Health Survey questionnaire [98]. This is a 36 question self-administered questionnaire used to measure functional health and well-being. Bowel habits and gastrointestinal feelings will be assessed at baseline, 12 and 24 weeks using our standard gastrointestinal feelings ratings form (abdominal comfort, bloating, flatulence, rumbling of stomach) and bowel habits questionnaire (number of bowel movements and Bristol Stool Chart).

\section{Oral glucose tolerance test (OGTT)}

Participants will consume a standardized menu the day prior to the OGTT to ensure consistency in macronutrient consumption prior to testing. The menu has been designed by a dietitian and includes 3 meals and 2 snacks. Participants will be instructed to finish dinner and then to not eat after $8 \mathrm{pm}$. The following morning, a baseline blood sample will be taken via a cannula inserted into the antecubital vein. Subsequently, participants will consume a $75 \mathrm{~g}$ oral glucose drink and blood samples will be drawn at 30, 60, 120 and 180 minutes. The OGTT will be conducted at baseline and follow-up (24 weeks). Blood will be centrifuged and plasma frozen at $-80{ }^{\circ} \mathrm{C}$ for future analyses. Glucose will be quantified using a glucose trinder assay (Stan Bio, Boerne, TX, USA) as per our previous work [72].

\section{Plasma lipids, liver biochemistry, and inflammatory markers}

At baseline, 12 and 24 weeks, a fasting blood sample will be taken for measurement of plasma HbA1c, lipids, CRP, liver enzymes (ALT, ALP, AST), FibroTest, and biochemical markers of liver function (creatinine, electrolytes, albumin, bilirubin). Blood will be drawn at and analysed by Calgary Laboratory Services (Calgary, AB, Canada). Serum adipokines and inflammatory markers (adiponectin, MCP-1, TNF- $\alpha$, IL- 6 and IL- 8 ) will be quantified using Milliplex kits (Millipore, Billerica, MA) from fasting samples obtained at baseline and follow-up (24 weeks) testing.

\section{Satiety hormones}

At each of the five time points during the OGTT, additional blood samples will be collected for measurement of satiety hormones including insulin. Blood will be drawn into a cooled EDTA vacutainer tube containing diprotinin-A $(0.034 \mathrm{mg} / \mathrm{ml}$ blood; MP Biomedicals, Irvine, CA); Sigma protease inhibitor $(1 \mathrm{mg} / \mathrm{ml}$ blood; Sigma Aldrich, Oakville, ON, Canada) and Roche Pefabloc (1 $\mathrm{mg} / \mathrm{ml}$ of blood; Roche, Mississauga, ON, Canada) [72]. Blood will be centrifuged within $30 \mathrm{~min}$ and plasma analyzed for ghrelin (active), insulin, leptin, glucose-dependent insulinotropic polypeptide (GIP) (total), GLP-1 (active), and PYY (total) concentrations using a Milliplex MAP Human Gut Hormone Panel Kit (Millipore, St Charles, MO). Surrogate markers of insulin resistance, HOMA and QUICKI, will be quantified as per our previous work [99].

\section{Mechanistic secondary outcomes \\ Gut Microbiota}

Stool will be collected at baseline and follow-up for analysis of gut microbiota. Participants will be instructed on proper methods for stool collection and all materials will be provided in a convenient specimen collection kit. Each subject will collect 2 tablespoons of stool into a pre-labeled sterile container. The container will be sealed, placed in a biohazard bag, and immediately stored in a standard home freezer $\left(-20^{\circ} \mathrm{C}\right)$. The specimen will be transported to our research lab within 4 days 
of collection in a Styrofoam container on an ice pack and transferred to $-80{ }^{\circ} \mathrm{C}$ for longer term storage. For analysis of fecal microbiota, total DNA will be extracted from $150 \mathrm{mg}$ of stool using the Qiagen QIAamp ${ }^{\mathrm{mm}}$ DNA Stool Mini Kit. Uniform bacterial DNA extraction is ensured by the addition of a 2-minute mechanical bead (0.1 $\mathrm{mm}$ zirconia:silica) beating step following addition of buffer ASL to the samples. DNA quantity and purity will be measured using a nanoscale spectrophotometer. To understand the functional and metabolic capabilities of the gut microbiota [100] and how prebiotics impact the metabolic capacity of the entire microbiome, whole genome shot-gun sequencing will be performed to analyze both the microbial composition and potential metabolic functions at the University of Alberta's Applied Genomic Center. Fecal DNA will be used as input for the Illumina Nextera XT DNA Sample Preparation Kit to construct indexed paired-end DNA libraries as previously described [101]. A final constructed pairedend indexed library set will be run on a Bioanalyzer 2100 using Agilent High Sensitivity DNA Kit to acquire library average size distribution (Agilent Technologies, Santa Clara). Final libraries will be quantified using a Qubit $^{\circ} 1.0$ fluorometer and the Qubit ${ }^{\circ}$ dsDNA HS assay (Life Technologies, Carlsbad). Obtained quantification and average size distribution of the final Nextera ${ }^{\circ}$ XT libraries will be used to calculate molarity of the library according to the Nextera Library Validation and Cluster Density Optimization Technical Note. Libraries are normalized to $2 \mathrm{nM}$ and pooled together using equal volume aliquots. The pooled and indexed library set is denatured, diluted, and sequenced on an Illumina MiSeq. Sequencing parameters consist of: paired-end 251 bp dual index sequencing chemistry using MiSeq Reagent Kit-v2 (500 cycle) and FASTQ Only workflow. Produced FASTQ files are then subjected to bioinformatics analysis. The raw FASTQ files are first filtered for adapter sequences and end trimmed of bases with quality less than 15. Read ends are then aligned against a set of mitochondrial databases with SOAPalign, with unaligned reads passed to each successive alignment. Read ends unaligned to mitochondrial databases are aligned to human, bacterial, and viral databases generated from NT, using SOAPalign. SpecI (http://vm-lux.embl.de/ $\sim$ kultima/MOCAT/) will be used to determine species composition and HUMAnN (http://huttenhower.sph.harvard.edu/humann) used for the characterization of microbial pathways in the communities.

\section{Endotoxin}

Serum endotoxin will be measured using a PyroGene recombinant factor $\mathrm{C}$ endotoxin detection kit (Lonza, Walkersville, MD) as per our previous work [75].

\section{Volatile organic compounds}

Functional capabilities of microbiota will be assessed in terms of bacterial production of VOC. At the time of analysis, fecal samples will be thawed and a carboxen/polydimethyl-siloxane solid phase micro-extraction (SPME) fiber will be placed into the sample collection vial by injecting it through the septum in the cap. The SPME fiber will be left in the vial headspace for 20 minutes to fully absorb the VOC. The fiber will then be retracted into the needle and transferred to the injector port for gas chromatographic (GC) analysis. Two GC machines connected in serial are used to resolve VOC that are detected by mass spectrometry (MS). Compounds are then identified by reference to the NIST 08 MS library.

\section{De Novo lipogenesis}

Energy reduction and weight loss significantly reduces lipogenesis, contributing to reduction in steatosis [102-106]. As part of our mechanistic investigation, fatty acid synthesis will be measured from fasting plasma samples using the deuterium incorporation method as per established methods [12, 107]. This protocol will be performed at a baseline visit prior to the OGTT (Fig. 1). Briefly, deuterium-labeled water $\left({ }^{2} \mathrm{H}_{2} \mathrm{O} ; 99.9\right.$ atom \%; Cambridge Isotope Laboratories, Inc.; Tewksbury, MA) will be provided to participants at a dose of $1 \mathrm{~g} / \mathrm{kg}$ body water (estimated as $60 \%$ of body weight) to ingest [107]. On day 1, a fasting blood sample will be taken to measure background ${ }^{2} \mathrm{H}$ enrichment, and on day 6, participants will ingest a single dose of ${ }^{2} \mathrm{H}_{2} \mathrm{O}$, as well as be provided with a second dose diluted in $1.5 \mathrm{~L}$ of water to consume at regular intervals over the next $24 \mathrm{~h}$ [107]. On day 7 at the inpatient visit, a fasting blood sample will be taken for measurement of ${ }^{2} \mathrm{H}$ incorporation into triglyceride (TG) and plasma water. Plasma will be ultracentrifugated to isolate total TG-rich lipoproteins, which at fasting will be primarily VLDL arising from the liver $[12,108]$. Samples will be stored at $-80{ }^{\circ} \mathrm{C}$ until further analysis. Folch extraction and thin layer chromatography will be used to isolate the TG portion, which will be saponified and methylated to prepare fatty acid methyl esters (FAME) for GC/P/IRMS analysis [107]. Samples will be analyzed for ${ }^{2} \mathrm{H}$ enrichment in major lipogenic fatty acids (14:0, 16:0, $16: 1,18: 0$, and 18:1). Proportion of newly-synthesized fatty acid will be calculated for each fatty acid using established equations [107].

\section{Sample size calculation}

Our sample size is based on the primary outcomes of hepatic injury and liver fat. For hepatic injury, we used pilot data from our lab to determine that a sample size of $n=19$ in each group would have $80 \%$ power to detect 
a difference in liver stiffness of $4.1 \mathrm{kPa}$ with FibroScan assuming a standard deviation of $4.0 \mathrm{kPa}$ and a 0.025 two-sided significance level. For the Fibrotest [109], a sample size of $n=10$ per group would have $80 \%$ power to detect a difference in Fibrotest score (0.0 to 1.0) of 0.08 assuming a standard deviation of 0.056 and a 0.025 two-sided significance level. For liver fat measured by MRI [110], a sample size of $n=25$ per group will have $80 \%$ power to detect a difference in hepatic fat of $4.4 \%$ assuming a standard deviation of $5.0 \%$ and a 0.025 twosided significance level. Based on these calculations, we will enroll $n=25$ per group. Assuming a dropout rate similar to that in our previous prebiotic trial [72] of approximately $20 \%$, we will recruit a total of 60 participants ( $n=30$ per group).

\section{Statistical analyses}

Statistical analyses will be performed using SPSS 22.0 software (IBM, Armonk, NY). Values with skewed distribution will be logarithmically transformed prior to analysis. Baseline characteristics will be compared between groups using chi-square for categorical variables and t-tests for continuous variables. Primary analysis will be performed on an intent-to-treat basis, regardless of subject compliance. The primary outcome measures are change from baseline to 24 weeks in hepatic injury (Fibrotest, FibroScan) and liver fat (percent measured by MRI). ANCOVA, with testing for confounding factors (sex) and potential covariates (BMI, age and fasting insulin) will be used to assess the difference between groups at weeks 0 and 24. Should the main effect of diet be significant, Tukey's multiple comparison test will be used. Secondary outcomes measured at 0 to 24 weeks (e.g. satiety hormones, body fat, glycemia and insulinemia) will be analyzed with ANCOVA as above. For variables measured over time (e.g. body weight, satiety hormones and glucose at $0,30,60,120$ and $180 \mathrm{mi}-$ nutes), a mixed model of repeated measures ANCOVA will be applied to examine the effect of treatment, time and their interaction. Our planned secondary analysis will be per protocol with all participants who complete the 24 week intervention and are compliant based on consumption of $\geq 80 \%$ the product doses. Cases with missing outcome data will be excluded from analysis. Results will be considered statistically significant (2sided) at $P \leq 0.025$ (Bonferroni adjustment for 2 primary outcomes).

Shotgun sequencing and GC-MS produce large datasets profiling microbiota composition and function and VOC metabolites, respectively. Binary as well as abundance data will be used in modeling VOC data. OPL-S coefficients determine the major variables contributing to discrimination in the model. VOC of interest will be identified with $90 \%$ probability by referencing to mass spectral library standards of the National Institute of Standards and Technology (NIST08) database. Bacterial communities will be quantitatively assessed by BrayCurtis dissimilarity matrix and Shannon-Wiener diversity index. Relative abundance data will be transformed $[\log (X+1)]$ prior to generation of the resemblance matrix. To reduce dimension of explanatory variables and identify bacteria that were potentially acting in groups we will conduct hierarchical clustering analysis using Spearman rank correlation with complete linkage and groups identified based on correlations $>0.5$. Distinct bacteria tax identified from hierarchical clustering analysis will be compared across groups and timepoints using formal parametric/non-parametric statistical methods such as the Dirichlet-multinomial distribution test, Mantel test, NP-Manova etc. We will analyze the prebiotic and placebo participants in terms of fecal microbiota, VOC, lipogenesis, clinical data, and host metabolic, nutritional, and inflammatory parameters using differential correlation analysis. Features will be compared using a Spearman's rank order correlation coefficient and correlations above a defined threshold will be compared between groups. We will then graph the correlations that are statistically different between the groups to identify the pairs of features (i.e. the different VOC metabolites and bacteria between treatments) that distinguish the responses. The network models will be visualized in the popular open-source Cytoscape software.

\section{Discussion}

The prevalence of NAFLD continues to increase worldwide in parallel with obesity and insulin resistance. Despite a multiplicity of factors working in concert to promote the development and progression of the disease, there is sufficient evidence linking NAFLD with dysbiosis of the gut microbiota to warrant an intervention aimed at prebiotic-mediated manipulation of the gut microbiota. Further support for the immediate need for this trial comes from the realization that there are few approved treatment options for NAFLD patients. While the standard of care for patients with NAFLD focuses on weight loss through diet and exercise, the difficulty in achieving and maintaining weight loss is well documented. Given that dietary prebiotic intake is associated with subjective improvements in satiety, there is the added potential for prebiotics to improve adherence to the weight loss intervention. Although approved therapies for NAFLD are still lacking, capitalizing on the gut microbiota-liver axis may provide new therapeutic targets in future.

The planned double blind, randomized clinical trial will determine whether adding prebiotic to a 6-month weight loss intervention will augment improvements in 
hepatic steatosis and fibrosis attributed to weight loss alone in the placebo group receiving an identical weight loss intervention but without supplementation. The exploration into the mechanisms of action is critically important in advancing treatment options for NAFLD and this study will specifically address de novo lipogenesis and metagenomic analysis of gut microbiota and their associated metabolic by-products (i.e. VOCs). Ultimately this clinical trial will advance our understanding of the magnitude to which NAFLD can be treated with a dietary intervention aimed at modulating the gut microbiota.

The proposed study design using daily packets to be consumed with water prior to a meal is feasible as indicated by our previous research [72] and is similar to soluble fiber supplements already on the market. More promising, however, is the versatility of prebiotics. Foods supplemented with prebiotics are already in production because the physical properties and sweet taste allows manufactures to decrease the caloric value while maintaining the palatability and texture of the food [111]. This ease of incorporation is important given the surge in interest in manipulation of the gut microbiota to prevent or treat conditions such as obesity and inflammatory bowel disease. While other options including fecal transplantation and antimicrobial-based interventions are being tested as viable means to manipulate the gut microbiota, targeted dietary interventions such as prebiotics or probiotics are easy to administer and for the most part have good safety and tolerance records [112]. While both prebiotics and probiotics are promising means to manipulate the gut microbiota, unlike prebiotics, probiotics must remain viable during storage and also be capable of surviving the harsh intestinal environment of the host in order to exert their beneficial effects [113]. Additionally, given the wellestablished health promoting properties of prebiotics and their tolerability, they could be used not only as a treatment but also as a prophylactic.

This trial will assess the ability of a prebiotic to improve overall health in a group at high-risk for a host of metabolic diseases. Improvement in liver health is expected, as are improvements in other predictors of the metabolic syndrome. Ultimately, the research will provide clinical evidence for a potential low-risk treatment option for NAFLD patients. Should the prebiotic intervention prove beneficial, it could have a significant impact on the ability of health professionals to recommend evidence-based targeted dietary interventions for managing NAFLD.

\section{Abbreviations}

ALP: alkaline phosphatase level; ALT: alanine aminotransferase;

ANCOVA: analysis of covariance; AST: aspartate aminotransferase; BMI: body mass index; CRP: C-reactive protein; DXA: dual energy x-ray absorptiometry; FAME: fatty acid methyl esters; GGT: gamma-glutamyl transpeptidase; GIP: glucose-dependent insulinotropic polypeptide; GLP-1: glucagon-like peptide-1; GC-MS: gas chromatography-mass spectroscopy;

HbA1c: glycosylated hemoglobin; HOMA-IR: homeostasis model assessment of insulin resistance; IL6: interleukin 6; IL-8: interleukin 8; LPS: lipopolysaccharide; MCP1: monocyte chemoattractant protein-1; NAFLD: non-alcoholic fatty liver disease; NAS: non-alcoholic fatty liver disease activity score; NASH: non-alcoholic steatohepatitis; MRI: magnetic resonance imaging; OGT: oral glucose tolerance test; PYY: peptide tyrosine tyrosine; QUICKI: quantitative insulin sensitivity check index; SCFA: short chain fatty acids; TG: triglycerides; TNFa: tumor necrosis factor alpha; TPN: total parenteral nutrition; VAS: visual analogue scales; VOC: volatile organic compounds.

\section{Competing interests}

JEL, JAP, BE, MR, MRB, KPR, KLM declare no conflict of interest. RAR previously held a research grant from the manufacturers of Synergy 1 prebiotic for a project unrelated to the current study.

\section{Authors' contributions}

JAP and JEL drafted the manuscript. JAP contributed to study design related to gut microbiota analysis and prebiotic dosing, and provided manuscript revisions. JEL contributed to study design and lipogenesis measurements. BE, MR and KPR contributed to study design and will be responsible for participant recruitment and monitoring. MRB contributed to study design and generation of pilot data. KLM contributed to study design and will perform shotgun sequencing for gut microbiota analyses. RAR conceived of the study, secured funding, was involved in the overall design, and helped to draft the manuscript. All authors read and approved the final manuscript.

\section{Acknowledgements}

This work is funded by a grant held by RAR from the Canadian Institutes for Health Research (MOP-136889). The funding agency had no role in the design of the study or preparation of this manuscript, and will have no influence on the data collection, analysis, and interpretation or manuscript publication. BE is supported by a Canadian Institutes of Health Research (CIHR) Signature Initiative Team Grant in Chronic Diseases. JEL was supported by a CIHR Postdoctoral Fellowship and University of Calgary Eye's High Postdoctoral Fellowship. MRB is supported by an Natural Sciences and Engineering Research Council of Canada Doctoral Studentship, an Alberta Innovates Health Solutions Doctoral Studentship and an Honorary Killam Doctoral Award.

\section{Author details}

${ }^{1}$ Faculty of Kinesiology, University of Calgary, 2500 University Dr. NW, Calgary, AB T2N 1N4, Canada. ${ }^{2}$ Health and Physical Education, Mount Royal University, 4825 Mount Royal Gate SW, Calgary, AB T3E 6K6, Canada. ${ }^{3}$ Snyder Institute for Chronic Diseases, Health Research and Innovation Center, University of Calgary, 3280 Hospital Drive NW, Calgary, AB T2N 4N1, Canada. ${ }^{4}$ Division of Gastroenterology and Hepatology, Department of Medicine, University of Calgary, 3280 Hospital Drive NW, Calgary, AB T2N 4N1, Canada. ${ }^{5}$ Department of Microbiology and Infectious Diseases, University of Calgary, 1863 Health Sciences Centre, 3330 Hospital Drive NW, Calgary, AB T2N 4N1, Canada. ${ }^{6}$ Division of Gastroenterology, Centre of Excellence for Gastrointestinal Inflammation and Immunity Research, 7-142 Katz GroupRexall Centre, University of Alberta, Edmonton, AB T6G 2C2, Canada. ${ }^{7}$ Department of Biochemistry \& Molecular Biology, Cumming School of Medicine, 3330 Hospital Drive NW, Calgary, AB T2N 4N1, Canada.

Received: 28 October 2015 Accepted: 25 November 2015

Published online: 03 December 2015

\section{References}

1. Yilmaz $Y$, Younossi ZM. Obesity-associated nonalcoholic fatty liver disease. Clin Liver Dis. 2014;18:19-31.

2. Barrera F, George J. The role of diet and nutritional intervention for the management of patients with NAFLD. Clin Liver Dis. 2014;18:91-112.

3. Williams CD, Stengel J, Asike MI, Torres DM, Shaw J, Contreras M, et al. Prevalence of nonalcoholic fatty liver disease and nonalcoholic 
steatohepatitis among a largely middle-aged population utilizing ultrasound and liver biopsy: a prospective study. Gastroenterology. 2011;140:124-31.

4. Browning JD, Szczepaniak LS, Dobbins R, Nuremberg P, Horton JD, Cohen $J C$, et al. Prevalence of hepatic steatosis in an urban population in the United States: impact of ethnicity. Hepatology. 2004:40:1387-95.

5. Bugianesi E, McCullough AJ, Marchesini G. Insulin resistance: a metabolic pathway to chronic liver disease. Hepatology. 2005;42:987-1000.

6. Mokdad AH, Ford ES, Bowman BA, Dietz WH, Vinicor F, Bales VS, et al. Prevalence of obesity, diabetes, and obesity-related health risk factors, 2001. JAMA. 2003:289:76-9.

7. Day CP, James OF. Steatohepatitis: A tale of two "hits"? Gastroenterology. 1998;114:842-5.

8. Conlon BA, Beasley JM, Aebersold K, Jhangiani SS, Wylie-Rosett J. Nutritional management of insulin resistance in nonalcoholic fatty liver disease (NAFLD). Nutrients. 2013;5:4093-114.

9. Duseja A, Chawla YK. Obesity and NAFLD: The role of bacteria and microbiota. Clin Liver Dis. 2014;18:59-71.

10. Koo SH. Nonalcoholic fatty liver disease: molecular mechanisms for the hepatic steatosis. Clin Mol Hepatol. 2013:19:210-5.

11. Lomonaco R, Ortiz-Lopez C, Orsak B, Webb A, Hardies J, Darland C, et al. Effect of adipose tissue insulin resistance on metabolic parameters and liver histology in obese patients with nonalcoholic fatty liver disease. Hepatology. 2012;55:1389-97.

12. Lambert JE, Ramos-Roman MA, Browning JD, Parks EJ. Increased de novo lipogenesis is a distinct characteristic of individuals with nonalcoholic fatty liver disease. Gastroenterology. 2014;146:726-35.

13. Fabbrini E, Mohammed BS, Magkos F, Korenblat KM, Patterson BW, Klein S. Alterations in adipose tissue and hepatic lipid kinetics in obese men and women with nonalcoholic fatty liver disease. Gastroenterology. 2008;134: 424-31.

14. Donnelly $\mathrm{KL}$, Smith $\mathrm{Cl}$, Schwarzenberg SJ, Jessurun J, Boldt MD, Parks EJ. Sources of fatty acids stored in liver and secreted via lipoproteins in patients with nonalcoholic fatty liver disease. J Clin Invest. 2005;115: 1343-51.

15. Rahimi RS, Landaverde C. Nonalcoholic fatty liver disease and the metabolic syndrome: clinical implications and treatment. Nutr Clin Pract. 2013;28:40-51.

16. Caldwell S, Argo C. The natural history of non-alcoholic fatty liver disease. Dig Dis. 2010;28:162-8

17. Said A. Non-alcoholic fatty liver disease and liver transplantation: outcomes and advances. World J Gastroenterol. 2013;19:9146-55.

18. Byrne CD, Olufadi R, Bruce KD, Cagampang FR, Ahmed MH. Metabolic disturbances in non-alcoholic fatty liver disease. Clin Sci (Lond). 2009;116:539-64.

19. Schindhelm RK, Dekker JM, Nijpels G, Bouter LM, Stehouwer CD, Heine RJ, et al. Alanine aminotransferase predicts coronary heart disease events: a 10year follow-up of the Hoorn Study. Atherosclerosis. 2007;191:391-6.

20. Targher G, Bertolini L, Rodella S, Zoppini G, Lippi G, Day C, et al. Nonalcoholic fatty liver disease is independently associated with an increased prevalence of chronic kidney disease and proliferative/laser-treated retinopathy in type 2 diabetic patients. Diabetologia. 2008:51:444-50

21. Ratziu V, Giral P, Jacqueminet S, Charlotte F, Hartemann-Heurtier A, Serfaty $\mathrm{L}$, et al. Rosiglitazone for nonalcoholic steatohepatitis: one-year results of the randomized placebo-controlled Fatty Liver Improvement with Rosiglitazone Therapy (FLIRT) trial. Gastroenterology. 2008;135:100-10.

22. Sanyal AJ, Chalasani N, Kowdley KV, McCullough A, Diehl AM, Bass NM, et al. Pioglitazone, vitamin E, or placebo for nonalcoholic steatohepatitis. N Engl J Med. 2010;362:1675-85.

23. Chalasani NP, Sanyal AJ, Kowdley KV, Robuck PR, Hoofnagle J, Kleiner $D E$, et al. Pioglitazone versus vitamin $E$ versus placebo for the treatment of non-diabetic patients with non-alcoholic steatohepatitis: PIVENS trial design. Contemp Clin Trials. 2009;30:88-96.

24. Hoofnagle $\mathrm{JH}$, Van Natta ML, Kleiner DE, Clark JM, Kowdley KV, Loomba R, et al. Vitamin $\mathrm{E}$ and changes in serum alanine aminotransferase levels in patients with non-alcoholic steatohepatitis. Aliment Pharmacol Ther. 2013; 38:134-43.

25. Promrat K, Lutchman G, Uwaifo Gl, Freedman RJ, Soza A, Heller T, et al. A pilot study of pioglitazone treatment for nonalcoholic steatohepatitis. Hepatology. 2004;39:188-96.

26. Mazzella N, Ricciardi LM, Mazzotti A, Marchesini G. The role of medications for the management of patients with NAFLD. Clin Liver Dis. 2014;18:73-89.

27. Asrih M, Jornayvaz FR. Diets and nonalcoholic fatty liver disease: the good and the bad. Clin Nutr. 2014:33:186-90.
28. Than NN, Newsome PN. Non-alcoholic fatty liver disease: when to intervene and with what. Clin Med. 2015:15:186-90.

29. Vilar-Gomez E, Martinez-Perez Y, Calzadilla-Bertot L, Torres-Gonzalez A, Gra-Oramas B, Gonzalez-Fabian L, et al. Weight loss via lifestyle modification significantly reduces features of nonalcoholic steatohepatitis. Gastroenterology. 2015;149:367-78.

30. Vos MB. Nutrition, nonalcoholic fatty liver disease and the microbiome: recent progress in the field. Curr Opin Lipidol. 2014;25:61-6.

31. Wieland A, Frank DN, Harnke B, Bambha K. Systematic review: microbial dysbiosis and nonalcoholic fatty liver disease. Aliment Pharmacol Ther. 2015;24

32. Gibson GR, Wang X. Regulatory effects of bifidobacteria on the growth of other colonic bacteria. J Appl Bacteriol. 1994;77:412-20.

33. Cano PG, Santacruz A, Trejo FM, Sanz Y. Bifidobacterium CECT 7765 improves metabolic and immunological alterations associated with obesity in high-fat diet-fed mice. Obesity (Sliver Spring). 2013;21:2310-21.

34. Henao-Mejia J, Elinav E, Jin C, Hao L, Mehal WZ, Strowig T, et al. Inflammasome-mediated dysbiosis regulates progression of NAFLD and obesity. Nature. 2012;482:179-85.

35. Spencer MD, Hamp TJ, Reid RW, Fischer LM, Zeisel SH, Fodor AA. Association between composition of the human gastrointestinal microbiome and development of fatty liver with choline deficiency. Gastroenterology. 2011;140:976-86

36. Zhu L, Baker SS, Gill C, Liu W, Alkhouri R, Baker RD, et al. Characterization of gut microbiomes in nonalcoholic steatohepatitis (NASH) patients: a connection between endogenous alcohol and NASH. Hepatology. 2013;57: 601-9.

37. Mouzaki M, Comelli EM, Arendt BM, Bonengel J, Fung SK, Fischer SE, et al. Intestinal microbiota in patients with nonalcoholic fatty liver disease. Hepatology. 2013;58:120-7.

38. Raman M, Ahmed I, Gillevet PM, Probert CS, Ratcliffe NM, Smith S, et al. Fecal microbiome and volatile organic compound metabolome in obese humans with nonalcoholic fatty liver disease. Clin Gastroenterol Hepatol. 2013;11:868-75.

39. Wong W, Tse CH, Lam TT, Wong GL, Chim AM, Chu WC, et al. Molecular characterization of the fecal microbiota in patients with nonalcoholic steatohepatitis - a longitudinal study. PLoS One. 2013;8, e62885.

40. Ma YY, Li L, Yu CH, Shen Z, Chen LH, Li YM. Effects of probiotics on nonalcoholic fatty liver disease: a meta-analysis. World J Gastroenterol. 2013; 19:6911-8.

41. Ramakrishna BS. Role of the gut microbiota in human nutrition and metabolism. J Gastroenterol Hepatol. 2013;28 Suppl 4:9-17.

42. Ley RE, Bäckhed F, Turnbaugh P, Lozupone CA, Knight RD, Gordon J. Obesity alters gut microbial ecology. Proc Natl Acad Sci U S A. 2005;102: 11070-5.

43. Bäckhed F, Ding H, Wang T, Hooper LV, Koh GY, Nagy A, et al. The gut microbiota as an environmental factor that regulates fat storage. Proc Natl Acad Sci U S A. 2004;101:15718-23.

44. Karlsson F, Tremaroli V, Nielsen J, Bäckhed F. Assessing the human gut microbiota in metabolic diseases. Diabetes. 2013;62:3341-9.

45. Kalliomäki M, Collado MC, Salminen S, Isolauri E. Early differences in fecal microbiota composition in children may predict overweight. Am J Clin Nutr. 2008:87:534-8

46. Schwiertz A, Taras D, Schäfer K, Beijer S, Bos NA, Donus C, et al. Microbiota and SCFA in lean and overweight healthy subjects. Obesity (Silver Spring). 2010;18:190-5.

47. Zhang H, DiBaise JK, Zuccolo A, Kudrna D, Braidotti M, Yu Y, et al. Human gut microbiota in obesity and after gastric bypass. Proc Natl Acad Sci U S A. 2009;106:2365-70.

48. Musso G, Gambino R, Cassader M. Gut microbiota as a regulator of energy homeostasis and ectopic fat deposition: mechanisms and implications for metabolic disorders. Curr Opin Lipidol. 2010;21:76-83.

49. Turnbaugh PJ, Ley RE, Mahowald MA, Magrini V, Mardis ER, Gordon J. An obesity-associated gut microbiome with increased capacity for energy harvest. Nature. 2006;444:1027-31

50. Bäckhed F, Manchester JK, Semenkovich CF, Gordon J. Mechanisms underlying the resistance to diet-induced obesity in germ-free mice. Proc Natl Acad Sci U S A. 2007;104:979-84.

51. Cani PD, Amar J, Iglesias MA, Poggi M, Knauf C, Bastelica D, et al. Metabolic endotoxemia initiates obesity and insulin resistance. Diabetes. 2007;56: $1761-72$ 
52. Cani PD, Bibiloni R, Knauf C, Waget A, Neyrinck AM, Delzenne NM, et al. Changes in gut microbiota control metabolic endotoxemia-induced inflammation in high-fat diet-induced obesity and diabetes in mice. Diabetes. 2008;57:1470-81.

53. Cani PD, Possemiers S, Van de Wiele T, Guiot Y, Everard A, Rottier O, et al. Changes in gut microbiota control inflammation in obese mice through a mechanism involving GLP-2-driven improvement of gut permeability. Gut. 2009:58:1091-103.

54. Farhadi A, Gundlapalli S, Shaikh M, Frantzides C, Harrell L, Kwasny MM, et al. Susceptibility to gut leakiness: a possible mechanism for endotoxaemia in non-alcoholic steatohepatitis. Liver Int. 2008;28:1026-33.

55. Alisi A, Manco M, Devito R, Piemonte F, Nobili V. Endotoxin and plasminogen activator inhibitor-1 serum levels associated with nonalcoholic steatohepatitis in children. J Pediatr Gastroenterol Nutr. 2010;50:645-9.

56. Sabaté JM, Jouët $P$, Harnois F, Mechler C, Msika S, Grossin M, et al. High prevalence of small intestinal bacterial overgrowth in patients with morbid obesity: a contributor to severe hepatic steatosis. Obes Surg. 2008;18:371-7.

57. Henao-Mejia J, Elinav E, Thaiss CA, Licona-Limon P, Flavell RA. Role of the intestinal microbiome in liver disease. J Autoimmun. 2013:46:66-73.

58. Garner CE, Smith S, de Lacy CB, White P, Spencer R, Probert CS, et al. Volatile organic compounds from feces and their potential for diagnosis of gastrointestinal disease. FASEB J. 2007;21:1675-88.

59. Roberfroid MB. Prebiotics and probiotics: are they functional foods? Am J Clin Nutr. 2000;71(6 Suppl):1682S-7S.

60. Delzenne NM, Neyrinck AM, Cani PD. Gut microbiota and metabolic disorders: how prebiotic can work? Br J Nutr. 2013;109(Suppl):S81-5.

61. Parnell JA, Raman M, Rioux KP, Reimer RA. The potential role of prebiotic fibre for treatment and management of non-alcoholic fatty liver disease and associated obesity and insulin resistance. Liver Int. 2012;32:701-11.

62. Roberfroid M. Functional food concept and its application to prebiotics. Dig Liver Dis. 2002;34 Suppl 2:S105-10.

63. Roberfroid MB. Inulin-type fructans: functional food ingredients. J Nutr. 2007;137(11 Suppl):2493S-502S.

64. Daubioul C, Rousseau N, Demeure R, Gallez B, Taper H, Declerck B, et al. Dietary fructans, but not cellulose, decrease triglyceride accumulation in the liver of obese Zucker fa/fa rats. J Nutr. 2002;132:967-73.

65. Daubioul CA, Taper HS, De Wispelaere LD, Delzenne NM. Dietary oligofructose lessens hepatic steatosis, but does not prevent hypertriglyceridemia in obese zucker rats. J Nutr. 2000;130:1314-9.

66. Fiordaliso M, Kok N, Desager JP, Goethals F, Deboyser D, Roberfroid M, et al. Dietary oligofructose lowers triglycerides, phospholipids and cholesterol in serum and very low density lipoproteins of rats. Lipids. 1995;30:163-7.

67. Sugatani J, Wada T, Osabe M, Yamakawa K, Yoshinari K, Miwa M. Dietary inulin alleviates hepatic steatosis and xenobiotics-induced liver injury in rats fed a high-fat and high-sucrose diet: association with the suppression of hepatic cytochrome P450 and hepatocyte nuclear factor 4a expression. Drug Metab Dispos. 2006;34:1677-87.

68. Maurer AD, Chen Q, McPherson C, Reimer RA. Changes in satiety hormones and expression of genes involved in glucose and lipid metabolism in rats weaned onto diets high in fibre or protein reflect susceptibility to increased fat mass in adulthood. J Physiol. 2009;587(Pt 3):679-91.

69. Parnell JA, Reimer RA. Prebiotic fibres dose-dependently increase satiety hormones and alter Bacteroidetes and Firmicutes in lean and obese JCR:LA-cp rats. Br J Nutr. 2012;107:601-13.

70. Pyra KA, Saha DC, Reimer RA. Prebiotic fiber increases hepatic acetyl CoA carboxylase phosphorylation and suppresses glucose-dependent insulinotropic polypeptide secretion more effectively when used with metformin in obese rats. J Nutr. 2012;142:213-20.

71. Reimer RA, Maurer AD, Eller LK, Hallam MC, Shaykhutdinov R, Vogel HJ, et al. Satiety hormone and metabolomic response to an intermittent high energy diet differs in rats consuming long-term diets high in protein or prebiotic fiber. J Proteome Res. 2012;11:4065-74.

72. Parnell JA, Reimer RA. Weight loss during oligofructose supplementation is associated with decreased ghrelin and increased peptide $Y Y$ in overweight and obese adults. Am J Clin Nutr. 2009;89:1751-9.

73. Dehghan P, Pourghassem Gargari B, Asghari J-a M. Oligofructose-enriched inulin improves some inflammatory markers and metabolic endotoxemia in women with type 2 diabetes mellitus: a randomized controlled clinical trial. Nutrition. 2014;30:418-23

74. Maurer AD, Reimer RA. Maternal consumption of high-prebiotic fibre or -protein diets during pregnancy and lactation differentially influences satiety hormones and expression of genes involved in glucose and lipid metabolism in offspring in rats. Br J Nutr. 2011;105:329-38.

75. Bomhof MR, Saha DC, Reid DT, Paul HA, Reimer RA. Combined effects of oligofructose and Bifidobacterium animalis on gut microbiota and glycemia in obese rats. Obesity (Sliver Spring). 2014;22:763-71.

76. Abete I, Astrup A, Martínez JA, Thorsdottir I, Zulet MA. Obesity and the metabolic syndrome: role of different dietary macronutrient distribution patterns and specific nutritional components on weight loss and maintenance. Nutr Rev. 2010;68:214-31.

77. Batra P, Das SK, Salinardi T, Robinson L, Saltzman E, Scott T, et al. Relationship of cravings with weight loss and hunger. Results from a 6 month worksite weight loss intervention. Appetite. 2013;69:1-7.

78. Rigaud D, Ryttig KR, Angel LA, Apfelbaum M. Overweight treated with energy restriction and a dietary fibre supplement: a 6-month randomized, double-blind, placebo-controlled trial. Int J Obes. 1990;14:763-9.

79. Ryttig KR, Tellnes G, Haegh L, Bøe E, Fagerthun H. A dietary fibre supplement and weight maintenance after weight reduction: a randomized, double-blind, placebo-controlled long-term trial. Int J Obes. 1989;13:165-71.

80. Kellow NJ, Coughlan MT, Reid CM. Metabolic benefits of dietary prebiotics in human subjects: a systematic review of randomised controlled trials. $\mathrm{Br} J$ Nutr. 2014;111:1147-61.

81. Dewulf EM, Cani PD, Claus SP, Fuentes S, Puylaert PG, Neyrinck AM, et al. Insight into the prebiotic concept: lessons from an exploratory, double blind intervention study with inulin-type fructans in obese women. Gut. 2013;62:1112-21.

82. Promrat K, Kleiner DE, Niemeier HM, Jackvony E, Kearns M, Wands JR, et al. Randomized controlled trial testing the effects of weight loss on nonalcoholic steatohepatitis. Hepatology. 2010;51:121-9.

83. Malaguarnera M, Vacante M, Antic T, Giordano M, Chisari G, Acquaviva R, et al. Bifidobacterium longum with fructo-oligosaccharides in patients with non alcoholic steatohepatitis. Dig Dis Sci. 2012;57:545-53.

84. Mazhar SM, Shiehmorteza M, Sirlin CB. Noninvasive assessment of hepatic steatosis. Clin Gastroenterol Hepatol. 2009;7:135-40

85. Daubioul CA, Horsmans $Y$, Lambert P, Danse E, Delzenne NM. Effects of oligofructose on glucose and lipid metabolism in patients with nonalcoholic steatohepatitis: results of a pilot study. Eur J Clin Nutr. 2005;59:723-6.

86. Festi $D$, Schiumerini R, Marzi L, Di Biase AR, Mandolesi D, Montrone L, et al. Review article: The diagnosis of non-alcoholic fatty liver disease - availability and accuracy of non-invasive methods. Aliment Pharmacol Ther. 2013;37: 392-400.

87. Wong W, Vergniol J, Wong GL, Foucher J, Chan HL, Le Bail B, et al. Diagnosis of fibrosis and cirrhosis using liver stiffness measurement in nonalcoholic fatty liver disease. Hepatology. 2010;51:454-62.

88. Ratziu V, Massard J, Charlotte F, Messous D, Imbert-Bismut F, Bonyhay L, et al. Diagnostic value of biochemical markers (FibroTest-FibroSURE) for the prediction of liver fibrosis in patients with non-alcoholic fatty liver disease. BMC Gastroenterol. 2006;6:6.

89. Singh D, Das CJ, Baruah MP. Imaging of non alcoholic fatty liver disease: a road less travelled. Indian J Endocrinol Metab. 2013;17:990-5.

90. Reeder SB, Sirlin CB. Quantification of liver fat with magnetic resonance imaging. Magn Reson Imaging Clin N Am. 2010;18:337-57.

91. Godin G, Shephard RJ. Godin leisure-time exercise questionnaire. Med Sci Sports Exerc. 1997;26:S36-8

92. Yang C, Hsu Y. A review of accelerometry-based wearable motion detectors for physical activity monitoring. Sensors. 2010;10:7772-88.

93. Meredith-Jones K, Williams S, Galland B, Kennedy G, Taylor R. 24 h Accelerometry: impact of sleep-screening methods on estimates of sedentary behaviour and physical activity while awake. J Sports Sci. 2015;21.

94. Sasaki JE, John D, Freedson PS. Validation and comparison of ActiGraph activity monitors. J Sci Med Sport. 2011;14:411-6.

95. Cole RJ, Kripke DF, Gruen W, Mullaney DJ, Gillin JC. Automatic sleep/wake identification from wrist activity. Sleep. 1992;15:461-9.

96. Stubbs RJ, Hughes DA, Johnstone AM, Rowley E, Reid C, Elia M, et al. The use of visual analogue scales to assess motivation to eat in human subjects: a review of their reliability and validity with an evaluation of new hand-held computerized systems for temporal tracking of appetite ratings. $\mathrm{Br} J$ Nutr. 2000:84:405-15.

97. Blundell J, de Graaf C, Hulshof T, Jebb S, Livingstone B, Lluch A, et al. Appetite control: methodological aspects of the evaluation of foods. Obes Rev. 2010;11:251-70. 
98. David K, Kowdley KV, Unalp A, Kanwal F, Brunt EM, Schwimmer JB. Quality of life in adults with nonalcoholic fatty liver disease: baseline data from the nonalcoholic steatohepatitis clinical research network. Hepatology. 2009;49: 1904-12.

99. Grover GJ, Koetzner L, Wicks J, Gahler RJ, Lyon MR, Reimer RA, et al. Effects of the soluble fiber complex PolyglycopleX on glucose homeostasis and body weight in young Zucker diabetic rats. Front Pharmacol. 2011;2:47.

100. Le Chatelier E, Nielsen T, Qin J, Prifti E, Hildebrand F, Falony G, et al. Richness of human gut microbiome correlates with metabolic markers. Nature. 2013;500:541-6.

101. Law J, Jovel J, Patterson J, Ford G, O'keefe S, Wang W, et al. Identification of hepatotropic viruses from plasma using deep sequencing: A next generation diagnostic tool. PLoS One. 2013;8, e60595.

102. Schwarz JM, Neese RA, Turner S, Dare D, Hellerstein MK. Short-term alterations in carbohydrate energy intake in humans. Striking effects on hepatic glucose production, de novo lipogenesis, lipolysis, and whole-body fuel selection. J Clin Invest. 1995;96:2735-43.

103. Browning JD, Baker JA, Rogers T, Davis J, Satapati S, Burgess SC. Short-term weight loss and hepatic triglyceride reduction: evidence of a metabolic advantage with dietary carbohydrate restriction. Am J Clin Nutr. 2011;93: 1048-52.

104. Petersen KF, Dufour S, Befroy D, Lehrke M, Hendler RE, Shulman Gl. Reversal of nonalcoholic hepatic steatosis, hepatic insulin resistance, and hyperglycemia by moderate weight reduction in patients with type 2 diabetes. Diabetes. 2005;54:603-8

105. Sato $F$, Tamura $Y$, Watada $H$, Kumashiro $N$, Igarashi $Y$, Uchino $H$, et al. Effects of diet-induced moderate weight reduction on intrahepatic and intramyocellular triglycerides and glucose metabolism in obese subjects. J Clin Endocrinol Metab. 2007;92:3326-9.

106. Sevastianova K, Santos A, Kotronen A, Hakkarainen A, Makkonen J, Silander $K$, et al. Effect of short-term carbohydrate overfeeding and long-term weight loss on liver fat in overweight humans. Am J Clin Nutr. 2012;96:727-34.

107. Wilke MS, French MA, Goh YK, Ryan EA, Jones PJ, Clandinin MT. Synthesis of specific fatty acids contributes to VLDL-triacylglycerol composition in humans with and without type 2 diabetes. Diabetologia. 2009;52:1628-37.

108. Schneeman BO, Kotite L, Todd KM, Havel RJ. Relationships between the responses of triglyceride-rich lipoproteins in blood plasma containing apolipoproteins B-48 and B-100 to a fat-containing meal in normolipidemic humans. Proc Natl Acad Sci U S A. 1993;90:2069-73.

109. Ratziu V, De Ledinghen V, Oberti F, Mathurin P, Wartelle-Bladou C, Renou C, et al. A randomized controlled trial of high-dose ursodesoxycholic acid for nonalcoholic steatohepatitis. J Hepatol. 2011;54:1011-9.

110. Ryan MC, Itsiopoulos C, Thodis T, Ward G, Trost N, Hofferberth S, et al. The Mediterranean diet improves hepatic steatosis and insulin sensitivity in individuals with non-alcoholic fatty liver disease. J Hepatol. 2013;59:138-43.

111. Corradini C, Lantano C, Cavazza A. Innovative analytical tools to characterize prebiotic carbohydrates of functional food interest. Anal Bioanal Chem. 2013;405:4591-605.

112. Walsh CJ, Guinane CM, OToole PW, Cotter PD. Beneficial modulation of the gut microbiota. FEBS Lett. 2014;588:4120-30.

113. Vyas U, Ranganathan N. Probiotics, prebiotics, and synbiotics: gut and beyond. Gastroenterol Res Pract. 2012;8727:16

\section{Submit your next manuscript to BioMed Central and we will help you at every step:}

- We accept pre-submission inquiries

- Our selector tool helps you to find the most relevant journal

- We provide round the clock customer support

- Convenient online submission

- Thorough peer review

- Inclusion in PubMed and all major indexing services

- Maximum visibility for your research

Submit your manuscript at www biomedcentral com/submit 